\title{
Variational Iteration Algorithm-I with an Auxiliary Parameter for Solving Boundary Value Problems
}

\author{
Hijaz Ahmad ${ }^{1, *}$, Muhammad Rafiq ${ }^{1}$, Clemente Cesarano ${ }^{2}$ and Hulya Durur ${ }^{3}$ \\ ${ }^{1}$ Department of Mathematics, COMSATS Institute of Information Technology, \\ Wah Cantt, Pakistan \\ ${ }^{2}$ Section of Mathematics, International Telematic University Uninettuno, \\ Corso Vittorio Emanuele II, 39, 00186 Roma, Italy \\ ${ }^{3}$ Department of Computer Engineering, Faculty of Engineering, Ardahan University, \\ Ardahan, 75000, Turkey \\ *Corresponding author e-mail: hijaz555@gmail.com
}

\begin{abstract}
In this article, the variational iteration algorithm-I with an auxiliary parameter (VIA-I with AP) is elaborated to initial and boundary value problems. The effectiveness, absence of difficulty and accuracy of the proposed method is remarkable and its tractability is well suitable for the use of these type of problems. Some examples have been given to show the effectiveness and utilization of this technique. A comparison of variational iteration algorithm-I (VIA-I) along VIA-I with AP has been carried out. It can be seen that this technique is more appropriate than as VIA-I.
\end{abstract}

\section{Introduction}

The study of nonlinear and physical sciences presents various numerical and analytical techniques. Most of these techniques have deficiencies in connection with

Received: December 6, 2019; Accepted: February 10, 2020

2010 Mathematics Subject Classification: 65-XX.

Keywords and phrases: variational iteration algorithm-I, nonlinear and linear partial differential equations, VIA-I with an auxiliary parameter.

Copyright ( 2020 Hijaz Ahmad et al. This is an open access article distributed under the Creative Commons Attribution License, which permits unrestricted use, distribution, and reproduction in any medium, provided the original work is properly cited. 
their qualities. These deficiencies have huge and complex calculations, different results, limited convergence and not capable in most of the problems.

A Chinese mathematician [1] developed a very clear and fundamental building block of the variational iteration algorithm-I [2]. The Originator [3] himself advanced the technique to an excessive level. The fundamental characteristic of this technique is the nearness of the elements of adaptability and capacity in taking care of linear and nonlinear problems. This technique is free of the complications of Adomian's polynomials. No discretization, linearization and small parameter assumptions are required, which in actual ruins the physical nature of the problem. One of the fundamental qualities of this procedure is that approximate solution of incredible rightness can be acquired by just a couple of iterations. This strategy has a basic technique, satisfactory outcomes or more everything, it tends to be utilized in a wide class of models [4-6].

Hesameddini and Latifizadeh [7] developed a thought of variational iteration method dependent on Laplace transformation. Salkuyeh [8] demonstrated the intermingling of the variational iteration method for settling direct solution of ODEs with variable coefficients. Fractional initial value problems [9], ill-defined initial value problem [10], quadratic Riccati differential equation [11] and system of integro-differential [12, 13] all these need the use of this technique for powerful solutions. The system of boundary value problems of higher order can likewise be settled by variational iteration technique demonstrated by Noor and Mohyud-Din [13].

Every once in a while, alterations have been brought into the variational iteration technique. One of those alterations, Herişanu and Marinca's change [14] is very alluring. In this adjustment, this technique has been connected with the minimum squares innovation. Noor and Mohyud-Din [15] have improved the variational iteration method by combining it with the generalized Taylor series to advance the efficiency of this technique. A basic treatment for the calculation of nonlinear terms is utilized in [15]. Among those changes in the variational iteration method, Yilmaz and Inc's adjustment [16] is also valuable. Afterward, a new procedure has been introduced for finding the ideal estimation of the unknown auxiliary parameter [17].

In this paper, we solved some partial differential equations with boundary and initial value problems by variational iteration algorithm-I. 


\section{Variational Iteration Algorithm-I}

Consider a nonlinear differential equation

$$
L[u(x)]+N[u(x)]=c(x),
$$

where $L[u(x)]$ is a linear operator and $N[u(x)]$ is a non-linear term respectively, whereas $c(x)$ being source inhomogeneous term. Constructing a correctional function for Equ. (1) as,

$$
u_{k+1}(x)=u_{k}(x)+\int_{0}^{x} \lambda(\eta)\left[L\left\{u_{k}(\eta)\right\}+N\left\{\tilde{u}_{k}(\eta)\right\}-c(\eta)\right] d \eta
$$

where $\lambda(\eta)$ is a general Lagrange multiplier [19].

Applying the variation $\delta$ on the one side as well as the other side of Equ. (2) with respect to $u_{k}(x)$,

$$
\delta u_{k+1}(x)=\delta u_{k}(x)+\delta \int_{0}^{x} \lambda(\eta)\left[L\left\{u_{k}(\eta)\right\}+N\left\{\tilde{u}_{k}(\eta)\right\}-c(\eta)\right] d \eta
$$

where $\tilde{u}_{k}(\eta)$ is a restricted term which means $\delta \tilde{u}_{k}(\eta)=0$.

Lagrange multiplier $\lambda(\eta)$ can be identified using optimality conditions. Let $k$ approach to infinity. An exact solution may be written as

$$
u(x)=\lim _{k \rightarrow \infty} u_{k}(x)
$$

In short, the variational iteration algorithm-I for (1) is

$$
\left\{\begin{array}{l}
u_{0}(x) \text { is an appropriate guess } \\
u_{k+1}(x)=u_{k}(x)+\int_{0}^{x} \lambda(\eta)\left[L\left\{u_{k}(\eta)\right\}+N\left\{u_{k}(\eta)\right\}-c(\eta)\right] d \eta \\
k=0,1,2,3, \ldots
\end{array}\right.
$$

This algorithm is called variational iteration algorithm-I (VIA-I ) and can be used for finding the accurate solution of various problems. The modification of this method has been presented in a lot of problems, arising in numerous fields of applied sciences and engineering [20-27], to get the approximate and exact solution of nonlinear problems. 


\section{VIA-I with an Auxiliary Parameter}

In this section, an auxiliary parameter, say $h$, is suggested to use with VIM-I, which will improve the accuracy and efficiency of algorithm. So equation (5) involving $h$ may be written as

$$
\left\{\begin{array}{l}
u_{0}(x) \text { being an initial guess, } \\
u_{1}(x, h)=u_{0}(x)+h \int_{0}^{x} \lambda(\eta)\left[L\left\{u_{0}(\eta)\right\}+N\left\{u_{0}(\eta)\right\}-c(\eta)\right] d \eta, \\
u_{k+1}(x, h)=u_{k}(x, h)+h \int_{0}^{x} \lambda(\eta)\left[L\left\{u_{k}(\eta, h)\right\}+N\left\{u_{k}(\eta, h)\right\}-c(\eta, h)\right] d \eta \\
k=1,2,3, \ldots
\end{array}\right.
$$

This algorithm is said to be VIA-I with an auxiliary parameter. An important characteristic of this method is that, it has a lesser size of calculation, nor tough to investigate but have a capability to approximate the solution precisely in domain of wide range. Interested readers are encouraged to study the convergence of this technique in [28].

\section{Applications}

In order to show the applicability of the VIA-I with AP, we solve nonlinear and linear partial differential equations. These equations are discussed very much in literature, that is why we are choosing to solve these examples.

\section{Burgers' Equation}

Consider a homogeneous nonlinear PDE [29, 30]:

$$
\frac{\partial u}{\partial t}-u \frac{\partial u}{\partial x}-\frac{\partial^{2} u}{\partial x^{2}}=0
$$

with conditions

$$
\begin{gathered}
u(x, 0)=1-x, \\
u(0, t)=\frac{1}{1+t}, \\
u(1, t)=0,
\end{gathered}
$$


has exact solution

$$
u(x, t)=\frac{1-x}{1+t} .
$$

First, we solve this nonlinear PDE by VIA-I.

So, the correction functional for (7) becomes

$$
u_{k+1}(x, t)=u_{k}(x, t)+\int_{0}^{t} \lambda(\eta)\left\{\frac{\partial u_{k}(x, \eta)}{\partial \eta}-u_{k} \frac{\partial \tilde{u}_{k}(x, \eta)}{\partial x}-\frac{\partial^{2} \tilde{u}_{k}(x, \eta)}{\partial x^{2}}\right\} d \eta .
$$

Taking the variation $\delta$ w.r.t $u_{k}(x, t)$ on both sides of equation (9)

$$
\delta u_{k+1}(x, t)=\delta u_{k}(x, t)+\delta \int_{0}^{t} \lambda(\eta)\left\{\frac{\partial u_{k}(x, \eta)}{\partial \eta}-u_{k}(x, \eta) \frac{\partial \tilde{u}_{k}(x, \eta)}{\partial x}-\frac{\partial^{2} \tilde{u}_{k}(x, \eta)}{\partial x^{2}}\right\} d \eta
$$

Ignoring the restricted terms

$$
\begin{aligned}
\delta u_{k+1}(x, t) & =\delta u_{k}(x, t)+\delta \int_{0}^{t} \lambda(\eta)\left\{\frac{\partial u_{k}(x, \eta)}{\partial \eta}\right\} d \eta \\
& =\delta u_{k}(x, t)+\lambda(\eta) \delta u_{k}(x, t)-\int_{0}^{t} \lambda^{\prime}(\eta) \delta u_{k}(x, \eta) d \eta \\
& =(1+\lambda(\eta)) \delta u_{k}(x, t)-\int_{0}^{t} \lambda^{\prime}(\eta) \delta u_{k}(x, \eta) d \eta .
\end{aligned}
$$

The stationary conditions are:

$$
\begin{gathered}
\lambda^{\prime}(\eta)=0, \\
1+\lambda(\eta)=0,
\end{gathered}
$$

we obtain the value of $\lambda(\eta)$ which is $\lambda(\eta)=-1$.

Putting this value of $\lambda(\eta)$ in equation (9),

$$
u_{k+1}(x, t)=u_{k}(x, t)-\int_{0}^{t}\left\{\frac{\partial u_{k}(x, \eta)}{\partial \eta}-u_{k}(x, \eta) \frac{\partial u_{k}(x, \eta)}{\partial x}-\frac{\partial^{2} u_{k}(x, \eta)}{\partial x^{2}}\right\} d \eta \text {. }
$$


Initializing with

$$
u_{0}(x, t)=1-x
$$

other iterations can be find by using (10),

$$
\begin{aligned}
& u_{1}(x, t)=1-x+x t-t \\
& u_{2}(x, t)=1-x-t+x t-\frac{1}{3} t^{3}+\frac{1}{3} t^{3} x+t^{2}-t^{2} x, \\
& u_{3}(x, t)=\frac{t^{7}-7 t^{6}+21 t^{5}-42 t^{4}+63 t^{3}-63 t^{2}+63 t-63}{63}+(x-1),
\end{aligned}
$$

stopped at $u_{6}(x, t)$.

The absolute error of $u_{6}(x, t)$ in the solution domain $(x, t) \in[0,1] \times[0,3]$ is shown in Figure 1.

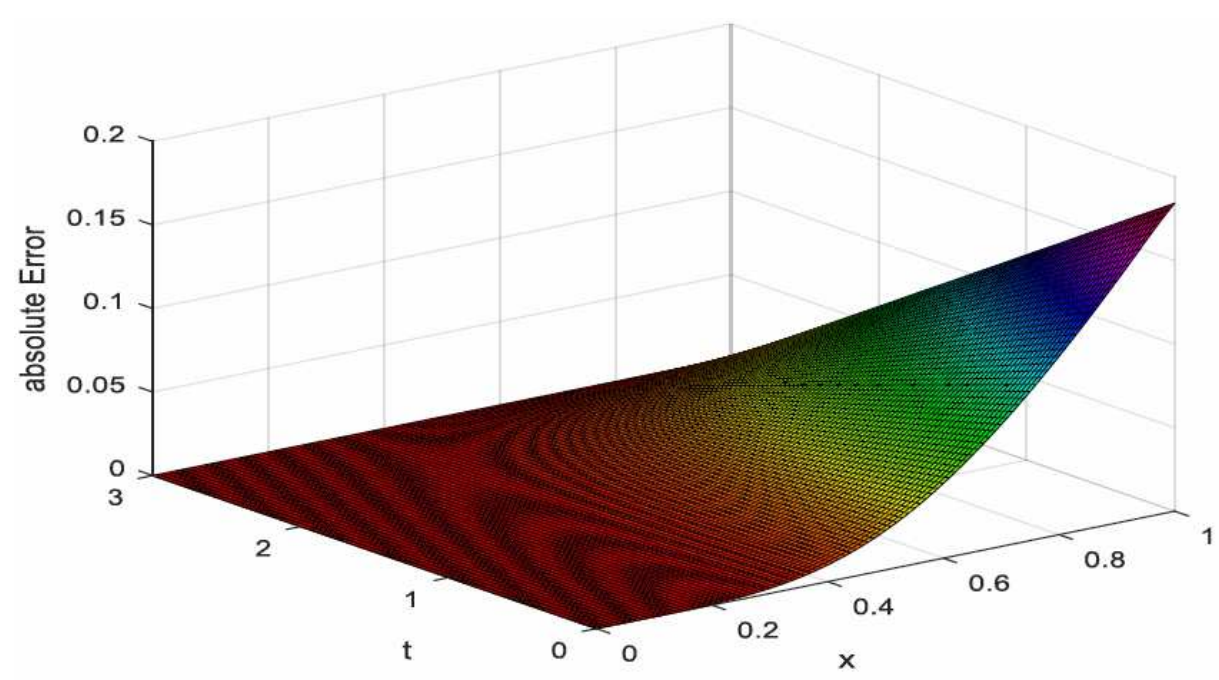

Figure 1. Absolute error between exact and approximate solutions by VIA-I.

In Figure 1, it is obvious that variational iteration algorithm-I diverges for big values of $t$. 
Next, the recurrence relation for VIA-I involving an auxiliary parameter is

$$
\begin{aligned}
u_{k+1}(x, t, h)= & u_{k}(x, t, h)-h \int_{0}^{t}\left\{\frac{\partial u_{k}(x, \eta, h)}{\partial \eta}\right. \\
& \left.-\left(u_{k}(x, \eta, h) \frac{\partial u_{k}(x, \eta, h)}{\partial x}\right)-\frac{\partial^{2} u_{k}(x, \eta, h)}{\partial x^{2}}\right\} d \eta .
\end{aligned}
$$

Starting with

$$
u_{0}(x, t)=1-x
$$

the following iterations are listed using relation (11)

$$
\begin{aligned}
& u_{1}(x, t, h)=(h t-1)(x-1), \\
& u_{2}(x, t, h)=(h t-1)(x-1) \\
& u_{3}(x, t, h)=(h t-1)(x-1)-\frac{h t(x-1)\left(-h^{2} t^{2}+3 h t+3 h-3\right)}{3},
\end{aligned}
$$

we stop the procedure at $u_{6}(x, t, h)$.

Let the residual function may be defined as

$$
r_{6}(x, t, h)=\frac{\partial u_{6}(x, t, h)}{\partial t}-u_{6}(x, t, h) \frac{\partial u_{6}(x, t, h)}{\partial x}-\frac{\partial^{2} u_{6}(x, t, h)}{\partial x^{2}} .
$$

So, the square of residual function for 10th order approximation w.r.t $h$ for $(x, t) \in[0,1] \times[0,3]$ is

$$
\frac{1}{(11)^{2}} \sum_{i=0}^{10} \sum_{j=0}^{10}\left(r_{6}\left(\frac{i}{10}, \frac{3 j}{10}, h\right)\right)^{2}
$$

The least result will occur at $h=0.78151800607$.

Hence, the absolute error between approximate and exact solutions is shown in Figure 2. 


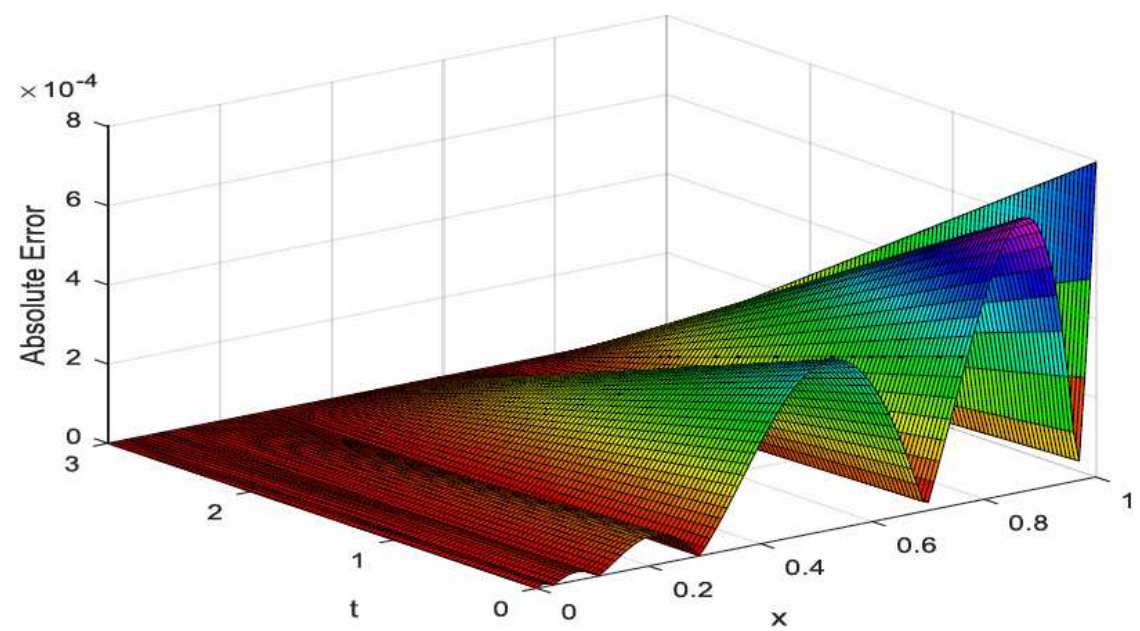

Figure 2. Absolute error between approximate and exact solutions by VIA-I with auxiliary parameter.

Comparing Figure 1 and Figure 2, it is clear that VIA-I with auxiliary parameter gives better results as compared to VIA-I.

Numerical comparison in both the approximate and exact solutions of both algorithms is given in Table 1.

Table 1. Comparison of absolute errors for 6th order approximation by VIA-I and VIA-I WITH AP.

\begin{tabular}{cccc}
\hline $\boldsymbol{x}$ & $\boldsymbol{t}$ & $\begin{array}{c}\text { Absolute Error in VIA-I } \\
\text { with AP }\end{array}$ & Absolute Error in VIA-I \\
\hline 0.1 & 0.3 & $8.449 \times 10^{-6}$ & $1.297 \times 10^{-5}$ \\
0.2 & 0.6 & $5.995 \times 10^{-5}$ & 0.000376 \\
0.3 & 0.9 & $1.269 \times 10^{-5}$ & 0.002092 \\
0.4 & 1.2 & 0.0001532 & 0.005988 \\
0.5 & 1.5 & 0.0002112 & 0.011790 \\
0.6 & 1.8 & 0.0001374 & 0.017900 \\
0.7 & 2.1 & $6.167 \times 10^{-6}$ & 0.021890 \\
0.8 & 2.4 & 0.0001018 & 0.021360 \\
0.9 & 2.7 & $6.486 \times 10^{-5}$ & 0.014490 \\
\hline
\end{tabular}

\section{A Nonlinear PDE}

Consider the homogeneous nonlinear PDE [29, 30]: 


$$
\frac{\partial u}{\partial t}-u-u \frac{\partial^{2} u}{\partial x^{2}}-\left(\frac{\partial u}{\partial x}\right)^{2}=0
$$

with conditions

$$
u(x, 0)=x^{\frac{1}{2}}, u(0, t)=0, u(1, t)=e^{t}
$$

has exact solution

$$
u(x, t)=x^{\frac{1}{2}} e^{t}
$$

First, we solve this nonlinear PDE by VIA-I.

So, the correction functional for (14) becomes

$$
\begin{aligned}
u_{k+1}(x, t)= & u_{k}(x, t)+\int_{0}^{t} \lambda(\eta)\left\{\frac{\partial u_{k}(x, \eta)}{\partial \eta}-\left(u_{k}(\tilde{x}, \eta)\right)\right. \\
& \left.-\left(u_{k}(x, \eta) \frac{\partial^{2} \tilde{u}_{k}(x, \eta)}{\partial x^{2}}\right)-\left(\frac{\partial \tilde{u}_{k}(x, \eta)}{\partial x}\right)^{2}\right\} d \eta .
\end{aligned}
$$

Taking the variation $\delta$ w.r.t $u_{k}(x, t)$ on both sides of equation (16)

$$
\begin{aligned}
\delta u_{k+1}(x, t)= & \delta u_{k}(x, t)+\delta \int_{0}^{t} \lambda(\eta)\left\{\frac{\partial u_{k}(x, \eta)}{\partial \eta}-\left(\tilde{u}_{k}(x, \eta)\right)\right. \\
& \left.-\left(u_{k}(x, \eta) \frac{\partial^{2} \tilde{u}_{k}(x, \eta)}{\partial x^{2}}\right)-\left(\frac{\partial \tilde{u}_{k}(x, \eta)}{\partial x}\right)^{2}\right\} d \eta .
\end{aligned}
$$

Ignoring the restricted terms

$$
\begin{aligned}
\delta u_{k+1}(x, t) & =\delta u_{k}(x, t)+\delta \int_{0}^{t} \lambda(\eta)\left\{\frac{\partial u_{k}(x, \eta)}{\partial \eta}\right\} d \eta \\
& =\delta u_{k}(x, t)+\lambda(\eta) \delta u_{k}(x, t)-\int_{0}^{t} \lambda^{\prime}(\eta) \delta u_{k}(x, \eta) d \eta \\
& =(1+\lambda(\eta)) \delta u_{k}(x, t)-\int_{0}^{t} \lambda^{\prime}(\eta) \delta u_{k}(x, \eta) d \eta
\end{aligned}
$$


The stationary conditions are:

$$
\begin{gathered}
\lambda^{\prime}(\eta)=0, \\
1+\lambda(\eta)=0,
\end{gathered}
$$

we get the value of $\lambda(\eta)$ which is $\lambda(\eta)=-1$.

Putting this value of $\lambda(\eta)$ in equation (16),

$$
\begin{aligned}
u_{k+1}(x, t)= & u_{k}(x, t)-\int_{0}^{t}\left\{\frac{\partial u_{k}(x, \eta)}{\partial \eta}-\left(u_{k}(x, \eta)\right)\right. \\
& \left.-\left(u_{k}(x, \eta) \frac{\partial^{2} u_{k}(x, \eta)}{\partial x^{2}}\right)-\left(\frac{\partial u_{k}(x, \eta)}{\partial x}\right)^{2}\right\} d \eta
\end{aligned}
$$

Starting with

$$
u_{0}(x, t)=x^{\frac{1}{2}}
$$

other iterations can be found by using the iterative scheme (17),

$$
\begin{aligned}
& u_{1}(x, t)=x^{\frac{1}{2}}(t+1), \\
& u_{2}(x, t)=x^{\frac{1}{2}}\left(\frac{\sqrt{t}+2 t+2}{2}\right), \\
& u_{3}(x, t)=x^{\frac{1}{2}}\left(\frac{t^{3}+3 t^{2}+6 t+6}{6}\right), \\
& u_{4}(x, t)=x^{\frac{1}{2}}\left(\frac{t^{4}+4 t^{3}+12 t^{2}+24 t+24}{24}\right), \\
& u_{5}(x, t)=x^{\frac{1}{2}}\left(\frac{t^{5}+5 t^{4}+20 t^{3}+60 t^{2}+120 t+120}{120}\right),
\end{aligned}
$$


we stop the procedure at $u_{10}(x, t)$.

The absolute error between approximate and exact solutions of $u_{10}(x, t)$ in domain $(x, t) \in[0,1] \times[0,4]$ is shown in Figure 3 .

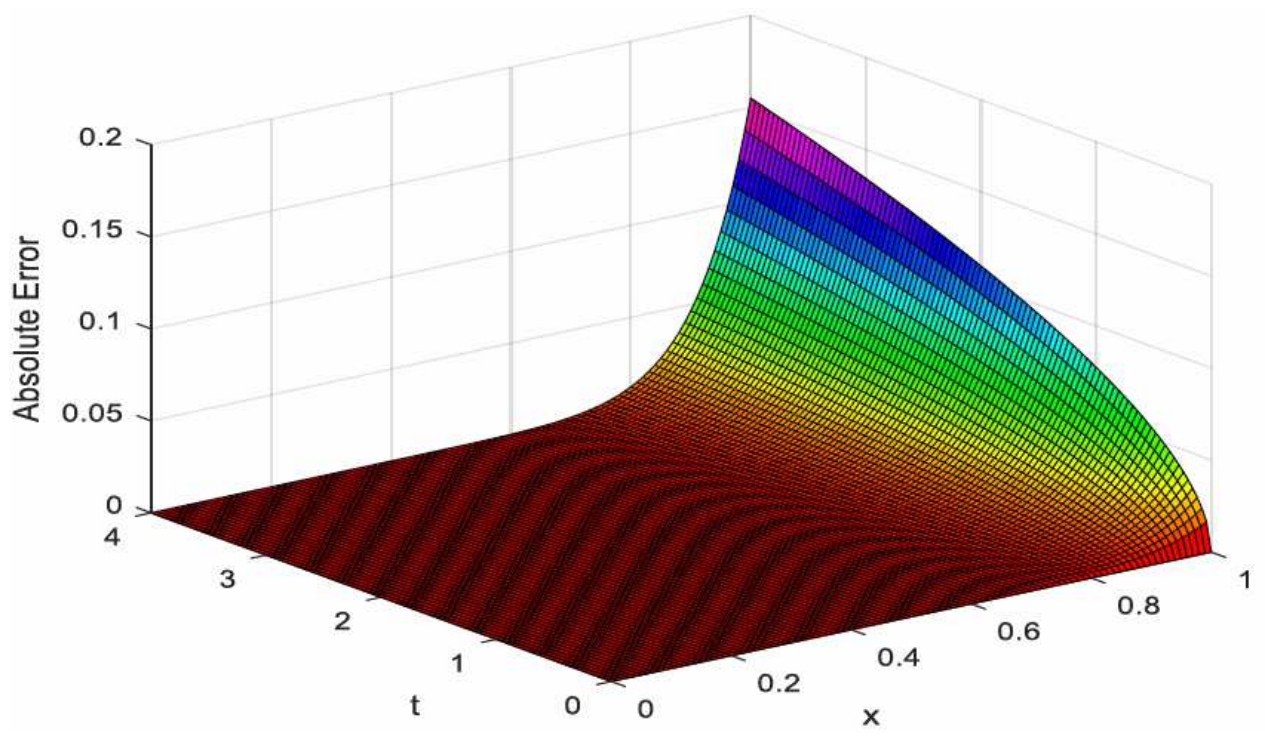

Figure 3. Absolute error between approximate and exact solution by VIA-I.

In Figure 3, it is obvious that variational iteration algorithm-I diverges for big values of $t$.

Next, the recurrence relation for VIA-I involving an auxiliary parameter is

$$
\begin{aligned}
u_{k+1}(x, t, h)= & u_{k}(x, t, h)-h \int_{0}^{t}\left\{\frac{\partial u_{k}(x, \eta, h)}{\partial \eta}-\left(u_{k}(x, \eta, h)\right)\right. \\
& \left.-\left(u_{k}(x, \eta, h) \frac{\partial^{2} u_{k}(x, \eta, h)}{\partial x^{2}}\right)-\left(\frac{\partial u_{k}(x, \eta, h)}{\partial x}\right)^{2}\right\} d \eta .
\end{aligned}
$$

Starting with

$$
u_{0}(x, t)=x^{\frac{1}{2}}
$$

the following iterations are listed using relation (18), 


$$
\begin{aligned}
& u_{1}(x, t, h)=x^{\frac{1}{2}}(h t+1) \\
& u_{2}(x, t, h)=x^{\frac{1}{2}}\left(\frac{h^{2} t^{2}-2 h^{2} t+4 h t+2}{2}\right) \\
& u_{3}(x, t, h)=x^{\frac{1}{2}}\left(\frac{h^{3} t^{3}-6 h^{3} t^{2}+6 h^{3} t+9 h^{2} t^{2}-18 h^{2} t+18 h t+6}{6}\right) \\
& u_{4}(x, t, h)=\frac{\left(x^{\frac{1}{2}}\right)}{24}\left(\begin{array}{c}
h^{4} t^{4}-12 h^{4} t^{3}+36 h^{4} t^{2}-24 h^{4} t+16 h^{3} t^{3} \\
-96 h^{3} t^{2}+96 h^{3} t+72 h^{2} t^{2}-14416 h^{2} t+96 h t+24
\end{array}\right)
\end{aligned}
$$

we stop the procedure at $u_{10}(x, t, h)$.

Let, the residual function may be defined as

$$
\begin{aligned}
r_{10}(x, t, h)= & \frac{\partial u_{10}(x, t, h)}{\partial \eta}-u_{10}(x, t, h) \\
& -u_{10}(x, t, h) \frac{\partial^{2} u_{10}(x, t, h)}{\partial x^{2}}-\left(\frac{\partial^{2} u_{10}(x, t, h)}{\partial x}\right)^{2} .
\end{aligned}
$$

The square of residual function for 10th order approximation w.r.t $h$ for $(x, t) \in[0,1] \times[0,4]$ is

$$
\frac{1}{(11)^{2}} \sum_{i=0}^{10} \sum_{j=0}^{10}\left(r_{10}\left(\frac{i}{10}, \frac{4 j}{10}, h\right)\right)^{2}
$$

The least result will occur at $h=1.1542483790$. Considering the value of $h$ in $u_{10}(x, t, h)$ for domain $(x, t) \in[0,1] \times[0,4]$, the absolute error between approximate and exact solution is shown in Figure 4. 


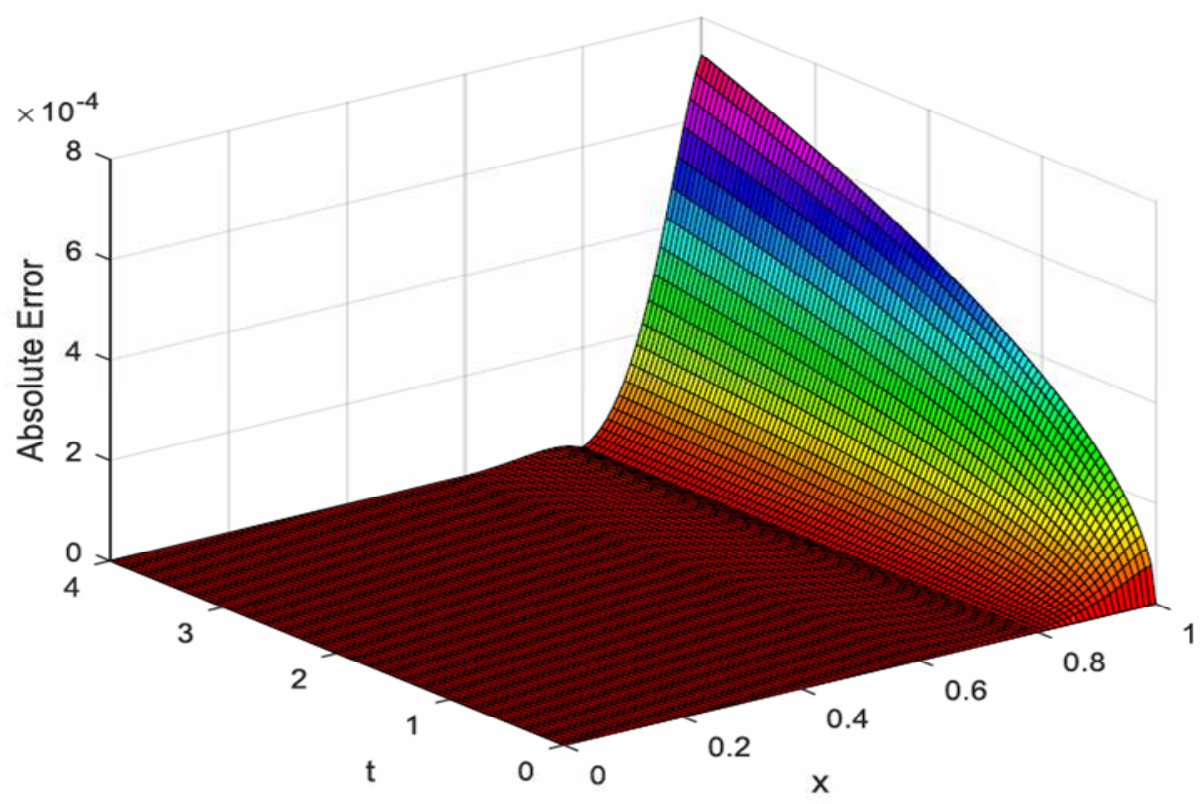

Figure 4. Absolute error between approximate and exact solution by VIA-I involving auxiliary parameter.

Comparing Figure 3 and Figure 4, it is clear that VIA-I involving auxiliary parameter reveals better performance rather than VIA-I .

So, a numerical comparison of absolute errors for both methods are shown in Table 2.

Table 2. A comparison of absolute errors for 10th order approximation by VIM- AI and VIA-I with auxiliary parameter.

\begin{tabular}{cccc}
\hline $\boldsymbol{x}$ & $\boldsymbol{t}$ & $\begin{array}{c}\text { Absolute error in VIA-I WITH } \\
\text { AP }\end{array}$ & Absolute error in VIA-I \\
\hline 0.1 & 0.4 & $1.648 \times 10^{-10}$ & $3.437 \times 10^{-13}$ \\
0.2 & 0.8 & $9.266 \times 10^{-10}$ & $1.031 \times 10^{-9}$ \\
0.3 & 1.2 & $4.625 \times 10^{-9}$ & $1.320 \times 10^{-7}$ \\
0.4 & 1.6 & $4.388 \times 10^{-8}$ & $3.211 \times 10^{-6}$ \\
0.5 & 2.0 & $2.691 \times 10^{-7}$ & $4.341 \times 10^{-5}$ \\
0.6 & 2.4 & $4.005 \times 10^{-7}$ & 0.003675 \\
0.7 & 2.8 & $7.944 \times 10^{-6}$ & 0.002253 \\
0.8 & 3.2 & $1.282 \times 10^{-6}$ & 0.010910 \\
0.9 & 3.6 & 0.0002083 & 0.044140 \\
1.0 & 4.0 & 0.0007266 & 0.155000 \\
\hline
\end{tabular}




\section{A Linear PDE}

Consider the homogeneous linear PDE [29, 30]:

$$
\frac{\partial u}{\partial t}+\frac{\partial u}{\partial x}-\frac{\partial^{2} u}{\partial x^{2}}=0
$$

with conditions

$$
\begin{gathered}
u(x, 0)=e^{x}-x \\
u(0, t)=1+t \\
\frac{\partial u}{\partial x}(0, t)=e-1
\end{gathered}
$$

has exact solution

$$
u(x, t)=t+e^{x}-x .
$$

First, we solve this linear PDE by variational iteration algorithm-I.

So the correction functional for equation (21) is

$$
u_{k+1}(x, t)=u_{k}(x, t)+\int_{0}^{t} \lambda(\eta)\left\{\frac{\partial u_{k}(x, \eta)}{\partial \eta}+\frac{\partial \tilde{u}_{k}(x, \eta)}{\partial x}-\frac{\partial^{2} \tilde{u}_{k}(x, \eta)}{\partial x^{2}}\right\} d \eta
$$

Taking the variation $\delta$ w.r.t $u_{k}(x, t)$ on both sides of the equation (23), we get

$$
\delta u_{k+1}(x, t)=\delta u_{k}(x, t)+\delta \int_{0}^{t} \lambda(\eta)\left\{\frac{\partial u_{k}(x, \eta)}{\partial \eta}+\frac{\partial \tilde{u}_{k}(x, \eta)}{\partial x}-\frac{\partial^{2} \tilde{u}_{k}(x, \eta)}{\partial x^{2}}\right\} d \eta
$$

Ignoring the restricted terms,

$$
\begin{aligned}
\delta u_{k+1}(x, t) & =\delta u_{k}(x, t)+\delta \int_{0}^{t} \lambda(\eta)\left\{\frac{\partial u_{k}(x, \eta)}{\partial \eta}\right\} d \eta \\
& =\delta u_{k}(x, t)+\lambda(\eta) \delta u_{k}(x, t)-\int_{0}^{t} \lambda^{\prime}(\eta) \delta u_{k}(x, \eta) d \eta \\
& =(1+\lambda(\eta)) \delta u_{k}(x, t)-\int_{0}^{t} \lambda^{\prime}(\eta) \delta u_{k}(x, \eta) d \eta .
\end{aligned}
$$


The stationary conditions are:

$$
\lambda^{\prime}(\eta)=0,1+\lambda(\eta)=0
$$

which gives $\lambda(\eta)=-1$.

Hence, the iterative scheme after using the value of $\lambda(\eta)$, may be written as

$$
u_{k+1}(x, t)=u_{k}(x, t)-\int_{0}^{t}\left\{\frac{\partial u_{k}(x, \eta)}{\partial \eta}+\frac{\partial u_{k}(x, \eta)}{\partial x}-\frac{\partial^{2} u_{k}(x, \eta)}{\partial x^{2}}\right\} d \eta
$$

Initializing with

$$
u_{0}(x, t)=t+e^{x}-x,
$$

other iterations can be obtained by using the iterative scheme (24),

$$
u_{1}(x, t)=t+e^{x}-x .
$$

Which is similar to equation (22), exact solution.

Applying VIA-I with an auxiliary parameter, then we can write

$$
\begin{aligned}
u_{k+1}(x, t, h)= & u_{k}(x, t, h) \\
& -h \int_{0}^{t}\left\{\frac{\partial u_{k}(x, \eta, h)}{\partial \eta}+\frac{\partial u_{k}(x, \eta, h)}{\partial x}-\frac{\partial^{2} u_{k}(x, \eta, h)}{\partial x^{2}}\right\} d \eta .
\end{aligned}
$$

Initializing with

$$
u_{0}(x, t)=u(x, 0)=e^{x}+x,
$$

other iterations can be obtained by using the relation (25),

$$
\begin{aligned}
& u_{0}(x, t)=u(x, 0)=e^{x}+x, \\
& u_{1}(x, t, h)=h t-x+e^{x}, \\
& u_{2}(x, t, h)=-t h^{2}+2 t h-x+e^{x}, \\
& u_{3}(x, t, h)=t h^{3}-3 t h^{2}+3 t h-x+e^{x},
\end{aligned}
$$




$$
\begin{aligned}
& u_{4}(x, t, h)=-t h^{4}+4 t h^{3}-6 t h^{2}+4 t h-x+e^{x}, \\
& u_{5}(x, t, h)=t h^{5}-5 t h^{4}+10 t h^{3}-10 t h^{2}+5 t h-x+e^{x},
\end{aligned}
$$

stopped at $u_{6}(x, t, h)$.

Thus residual function may be defined as

$$
r_{10}(x, t, h)=\frac{\partial u_{10}(x, t, h)}{\partial \eta}+\frac{\partial u_{10}(x, t, h)}{\partial x}-\frac{\partial^{2} u_{10}(x, t, h)}{\partial x^{2}} .
$$

The square of residual function for 10th order approximation w.r.t $h$ for $(x, t) \in[0,10] \times[0,10]$ is

$$
\frac{1}{(11)^{2}} \sum_{i=0}^{10} \sum_{j=0}^{10}\left(r_{10}\left(\frac{i}{10}, \frac{j}{10}, h\right)\right)^{2} .
$$

The least result will occur at $h=1.0$.

Consider this value in equation (25), we acquired

$$
\begin{aligned}
& u_{0}(x, t)=u(x, 0)=t+e^{x}+x, \\
& u_{1}(x, t, h)=t+e^{x}-x, \\
& u_{2}(x, t, h)=t+e^{x}-x, \\
& u_{3}(x, t, h)=t+e^{x}-x, \\
& u_{4}(x, t, h)=t+e^{x}-x, \\
& u_{5}(x, t, h)=t+e^{x}-x,
\end{aligned}
$$

which is the exact solution and it is the same result obtained by VIA-I in first iteration. 


\section{Conclusions}

In this paper, we have studied and analyzed two iterative methods, VIA-I with an auxiliary parameter and VIA-I. The presence of the elements of flexibility and capability in solutions of nonlinear equations is the very basic characteristic of VIA-I. One of the key characteristics of this method is that, exact solution or approximate solution of better accuracy can be gained by only a few iterations. Its other properties include the simple procedure of solution, acceptable results and above all, it can be practically utilized to a great number of nonlinear problems. The use of an auxiliary parameter $h$ in the VIA-I, the most favorable choice of it can remarkably improve the accuracy. The numerical and graphical results show that VIA-I with AP is a perfect and dependable technique for the solution of nonlinear and linear problems. The comparison shows that VIM-I including an auxiliary parameter presents efficient results in large domains as compared to VIA-I.

\section{References}

[1] J.-H. He, Variational iteration method - a kind of non-linear analytical technique: some examples, Int. J. Non-Linear Mech. 34(4) (1999), 699-708. https://doi.org/10.1016/S0020-7462(98)00048-1

[2] J.-H. He, G.-C. Wu and F. Austin, The variational iteration method which should be followed, Nonlinear Sci. Lett. A 1(1) (2010), 1-30.

[3] J.-H. He, Some asymptotic methods for strongly nonlinear equations, Int. J. Mod. Phys. B 20(10) (2006), 1141-1199. https://doi.org/10.1142/S0217979206033796

[4] M. Rafiq, H. Ahmad and S. T. Mohyud-Din, Variational iteration method with an auxiliary parameter for solving Volterra's population model, Nonlinear Sci. Lett. A 8(4) (2017), 389-396.

[5] H. Ahmad, Auxiliary parameter in the variational iteration algorithm-II and its optimal determination, Nonlinear Sci. Lett. A 9(1) (2018), 62-72.

[6] H. Ahmad, Variational iteration method with an auxiliary parameter for solving differential equations of the fifth order, Nonlinear Sci. Lett. A 9(1) (2018), 27-35.

[7] E. Hesameddini and H. Latifizadeh, Reconstruction of variational iteration algorithms using the Laplace transform, Int. J. Nonlinear Sci. Numer. Simul. 10(11-12) (2009), 13771382. https://doi.org/10.1515/IJNSNS.2009.10.11-12.1377

[8] D. K. Salkuyeh, Convergence of the variational iteration method for solving linear 
systems of ODEs with constant coefficients, Comput. Math. Appl. 56(8) (2008), 20272033. https://doi.org/10.1016/j.camwa.2008.03.030

[9] İ. Ateş and A. Yildirim, Application of variational iteration method to fractional initialvalue problems, Int. J. Nonlinear Sci. Numer. Simul. 10(7) (2009), 877-884. https://doi.org/10.1515/IJNSNS.2009.10.7.877

[10] C. Chun, Variational iteration method for a reliable treatment of heat equations with illdefined initial data, Int. J. Nonlinear Sci. Numer. Simul. 9(4) (2008), 435-440. https://doi.org/10.1515/IJNSNS.2008.9.4.435

[11] R. Mokhtari, Variational iteration method for solving nonlinear differential-difference equations, Int. J. Nonlinear Sci. Numer. Simul. 9(1) (2008), 19-24. https://doi.org/10.1515/IJNSNS.2008.9.1.19

[12] J. Saberi-Nadjafi and M. Tamamgar, The variational iteration method: a highly promising method for solving the system of integro-differential equations, Comput. Math. Appl. 56(2) (2008), 346-351. https://doi.org/10.1016/j.camwa.2007.12.014

[13] M. Aslam Noor and S. T. Mohyud-Din, Variational iteration technique for solving higher order boundary value problems, Appl. Math. Comput. 189(2) (2007), 1929-1942. https://doi.org/10.1016/j.amc.2006.12.071

[14] N. Herişanu and V. Marinca, A modified variational iteration method for strongly nonlinear problems, Nonlinear Sci. Lett. A 1(2) (2010), 183-192.

[15] M. A. Noor and S. T. Mohyud-Din, Modified variational iteration method for solving fourth-order boundary value problems, J. Appl. Math. Comput. 29(1-2) (2009), 81-94. https://doi.org/10.1007/s12190-008-0090-z

[16] E. Yilmaz and M. Inc, Numerical simulation of the squeezing flow between two infinite plates by means of the modified variational iteration method with an auxiliary parameter, Nonlinear Sci. Lett. A 1(3) (2010), 297-306.

[17] Hijaz Ahmad, Aly R. Seadawy and Tufail A. Khan, Numerical solution of Korteweg-de Vries-Burgers equation by the modified variational iteration algorithm-II arising in shallow water waves, Phys. Scr. 95(4) (2020). https://doi.org/10.1088/1402-4896/ab6070.

[18] M. A. Noor and S. T. Mohyud-Din, Variational homotopy perturbation method for solving higher dimensional initial boundary value problems, Math. Probl. Eng. 2008, Article ID 696734, 11 pp. https://doi.org/10.1155/2008/696734

[19] M. Inokuti, H. Sekine and T. Mura, General Use of the Lagrange Multiplier in Nonlinear Mathematical Physics, Pergamon Press, Oxford, 1978. 
[20] H. Ahmad and T. A. Khan, Variational iteration algorithm I with an auxiliary parameter for the solution of differential equations of motion for simple and damped mass-spring systems, Noise Vib. Worldw. 51(1-2) (2019), 12-20.

https://doi.org/10.1177/0957456519889958

[21] H. Ahmad, T. A. Khan and C. Cesarano, Numerical solutions of coupled Burgers' equations, Axioms 8(4) (2019), 119. https://doi.org/10.3390/axioms8040119

[22] H. Ahmad and T. A. Khan, Variational iteration algorithm-I with an auxiliary parameter for wave-like vibration equations, J. Low Freq. Noise Vib. Act. Control 38(3-4) (2019), 1113-1124,. https://doi.org/10.1177/1461348418823126

[23] M. Nadeem, F. Li and H. Ahmad, Modified Laplace variational iteration method for solving fourth-order parabolic partial differential equation with variable coefficients, Comput. Math. Appl. 78(6) (2019), 2052-2062.

https://doi.org/10.1016/j.camwa.2019.03.053

[24] H. Ahmad, Variational iteration algorithm-II with an auxiliary parameter and its optimal determination, Nonlinear Sci. Lett. A 9(1) (2018), 62-72.

[25] M. Nadeem and H. Ahmad, Variational iteration method for analytical solution of the Lane-Emden type equation with singular initial and boundary conditions, Earthline $J$. Math. Sci. 2(1) (2019), 127-142. https://doi.org/10.34198/ejms.2119.127142

[26] H. Ahmad, Variational iteration algorithm-I with an auxiliary parameter for solving Fokker-Planck equation, Earthline J. Math. Sci. 2(1) (2019), 29-37. https://doi.org/10.34198/ejms.2119.2937

[27] H. Ahmad, Variational iteration method with an auxiliary parameter for solving telegraph equations, J. Nonlinear Anal. Appl. 2018(2) (2018), 223-232.

https://doi.org/10.5899/2018/jnaa-00417

[28] H. Ghaneai and M. M. Hosseini, Variational iteration method with an auxiliary parameter for solving wave-like and heat-like equations in large domains, Comput. Math. Appl. 69(5) (2015), 363-373. https://doi.org/10.1016/j.camwa.2014.11.007

[29] M. A. Jafari and A. Aminataei, Improved homotopy perturbation method, Int. Math. Forum 5(32) (2010), 1567-1579.

[30] H. Kumar Mishra and A. K. Nagar, He-Laplace method for linear and nonlinear partial differential equations, J. Appl. Math. 2012, Article ID 180315, 16 pp.

https://doi.org/10.1155/2012/180315 